\title{
Vegetation-Plot Database of the University of the Basque Country (BIOVEG)
}

\author{
Idoia Biurrun, Itziar García-Mijangos, Juan A. Campos, Mercedes Herrera \& Javier \\ Loidi
}

\begin{abstract}
BIOVEG (Vegetation Database of the University of the Basque Country; GIVD ID EU-00-011) stores 20,172 phytosociological relevés mostly from the northern-central part of the Iberian Peninsula and southwestern France: Basque Country and surrounding areas. All vegetation types of this area are represented in the database: temperate deciduous, mediterranean evergreen and subalpine coniferous forests and related shrublands and grasslands, rock and scree vegetation, coastal vegetation, aquatic and semiaquatic vegetation and anthropogenic vegetation. Data are stored in TURBOVEG format, and are freely available under request. Many published relevés have also been included in SIVIM database (Information System of Iberian and Macaronesian Vegetation), and thus they are freely available online. Data have been mainly used for vegetation classification, but also for distribution analysis of species and communities.
\end{abstract}

Keywords: Northern Spain; phytosociological relevé; plot; Southern France.

\begin{tabular}{|c|c|}
\hline GIVD Database ID: EU-00-011 & Last update: $2012-07-09$ \\
\hline \multicolumn{2}{|c|}{ Vegetation-Plot Database of the University of the Basque Country (BIOVEG) } \\
\hline \multicolumn{2}{|c|}{ Scope: Phytosociological relevés of northern Iberian Peninsula and southern France } \\
\hline Status: completed and continuing & Period: $1941-2011$ \\
\hline \multicolumn{2}{|c|}{$\begin{array}{l}\text { Database manager(s): Idoia Biurrun (idoia.biurrun@ehu.es); Itziar García-Mijangos (itziar.garcia@ehu.es); Juan A. Campos } \\
\text { (juanan.campos@ehu.es) }\end{array}$} \\
\hline \multicolumn{2}{|c|}{ Owner: [NA] } \\
\hline \multicolumn{2}{|l|}{ Web address: $[N A]$} \\
\hline Availability: free upon request & Online search: no \\
\hline Database format(s): TURBOVEG & Export format(s): TURBOVEG, MS Access, Excel \\
\hline \multicolumn{2}{|l|}{ Publication: $[\mathrm{NA}]$} \\
\hline Plot type(s): normal plots & Plot-size range: $0,2-1,000 \mathrm{~m}^{2}$ \\
\hline Non-overlapping plots: 20,172 & Estimate of existing plots: $22,000 \quad$ Completeness: $92 \%$ \\
\hline Total plot observations: 20,172 & Number of sources: 200 \\
\hline \multicolumn{2}{|c|}{ Countries: ES: $98.0 \%$; FR: $1.9 \%$; PT: $0.1 \%$} \\
\hline \multicolumn{2}{|c|}{ Forest: $19 \%$ — Non-forest: aquatic: $2 \%$; semi-aquatic: $14 \%$; arctic-alpine: $2 \%$; natural: $6 \%$; semi-natural: $44 \%$; anthropogenic: $12 \%$} \\
\hline \multicolumn{2}{|c|}{ Guilds: all vascular plants: $100 \%$; bryophytes (terricolous or aquatic): $3 \%$} \\
\hline \multicolumn{2}{|c|}{ Environmental data: altitude: $98 \%$; slope aspect: $26 \%$; slope inclination: $48 \%$} \\
\hline \multicolumn{2}{|c|}{ Performance measure(s): presence/absence only: $0 \%$; cover: $100 \%$} \\
\hline \multicolumn{2}{|c|}{$\begin{array}{l}\text { Geographic localisation: GPS coordinates (precision } 25 \mathrm{~m} \text { or less): } 3 \% \text {; point coordinates less precise than GPS, up to } 1 \mathrm{~km}: 82 \% \text {; small grid } \\
\text { (not coarser than } 10 \mathrm{~km} \text { ): } 15 \%\end{array}$} \\
\hline $\begin{array}{l}\text { Sampling periods: } 1940-1949: 0.1 \\
0.1 \%\end{array}$ & 359: 0.4\%; 1960-1969: 1.7\%; 1970-1979: 31.2\%; 1980-1989: 35.8\%; 1990-1999: 9.8\%; 2000-2009: \\
\hline Information as of 20 & urther details and future updates available from http://www.givd.info/ID/EU-00-011 \\
\hline
\end{tabular}

Idoia Biurrun* (idoia.biurrun@ehu.es), Itziar García-Mijangos (itziar.garcia@ehu.es), Juan A. Campos (juanan.campos@ehu.es), Mercedes Herrera (meme.herrera@ehu.es), Javier Loidi (javier.loidi@ehu.es)

Plant Biology and Ecology, University of the Basque Country - UPV/EHU, P.O.Box 644, 48080 Bilbao, SPAIN

*Corresponding author 